\title{
Short-term Results of Magnetic Resonance Imaging after Ankle Distraction Arthroplasty
}

\author{
Amgad M Haleem ${ }^{1}$, Sherif Galal ${ }^{2}$, Ogonna K Nwawka ${ }^{3}$, Angela Balagadde ${ }^{4}$, Eugene W Borst ${ }^{5}$, Huong T Do ${ }^{6}$, Douglas N Mintz ${ }^{7}$, \\ Austin T Fragomen ${ }^{8}$, S Robert Rozbruch ${ }^{9}$
}

\begin{abstract}
Background: Ankle distraction arthroplasty has emerged as an alternative treatment for ankle arthritis. There are few reports on the magnetic resonance imaging (MRI) findings after distraction arthroplasty. This study sought to determine whether there are positive changes on MRI after ankle distraction and improvements on X-ray. Additionally, patient-reported outcomes and joint range of motion (ROM) after ankle distraction are described.

Materials and methods: Thirty-two patients (mean age 49 years) who underwent ankle distraction had pre-operative and one-year postoperative MRIs, which were graded using a modified whole-organ magnetic resonance imaging score (WORMS). Ankle joint space and ROM were measured. A non-validated three-item questionnaire was administered to assess functional outcomes.

Results: Although the anterior quadrant of the ankle showed a trend to improvement in cartilage morphology on the postoperative MRI, the WORMS did not demonstrate a significant difference in any of its subcategories. While reduction in joint osteophytes was observed and maintained short term, this was mainly due to resection intraoperatively. X-rays revealed a significant increase in joint space, and there was a significant increase in ankle dorsiflexion. Eight-seven percent of the patients were satisfied with their functional outcome.

Conclusion: At short-term follow-up, MRI scores after ankle distraction arthroplasty did not demonstrate significant improvement despite positive changes on X-ray and improved clinical outcomes and ankle ROM. Further study on larger patient numbers with longer follow-up is required. Level of evidence: IV, Case Series

Keywords: Ankle distraction, Clinical results, Magnetic resonance imaging.

Strategies in Trauma and Limb Reconstruction (2020): 10.5005/jp-journals-10080-1512
\end{abstract}

\section{INTRODUCTION}

Ankle arthritis remains a challenging problem, especially in the young, active patient population. Treatment options including ankle arthrodesis or total ankle arthroplasty are not without significant limitations. Arthrodesis, in the hands of fellowshiptrained foot and ankle surgeons, provides reliable postoperative pain relief, whereas loss of ankle motion for an active patient with a reasonable preoperative range of motion $(\mathrm{ROM})$ is a disadvantage. There is an increase in energy expenditure during ambulation as well as increased loads on adjacent hind foot joints leading to accelerated wear and potential risks of delayed and non-union., ${ }^{1,2}$ Total ankle replacement (TAR) provides pain relief while maintaining ankle ROM. Despite improved survivorship of newer generation implants, ${ }^{3}$ TAR remains technically demanding especially in ligamentous imbalance and severe deformities of the ankle. ${ }^{4}$ The finite life span of ankle prostheses hinders its use in younger active patients due to accelerated wear, osteolysis, loosening, and need for subsequent revisions with underlying bone loss.,

Ankle distraction arthroplasty is a surgical technique in which the tibiotalar surfaces are separated by an external fixator for a certain time period. ${ }^{7,8}$ It is valuable in patients with a preserved ROM or young age or both. The technique mechanically unloads the arthritic joint, optimises an inherent repair process, and may halt and possibly reverse the catabolic process of arthritis, leading to pain reduction while retaining $\mathrm{ROM} .^{9-13}$

Ankle distraction is hypothesised to relieve arthritic symptoms through a number of different mechanisms: mechanical unloading of the joint decreases abnormally high contact stresses on the
${ }^{1}$ Department of Orthopedic Surgery, University of Oklahoma Health Sciences Center, Oklahoma City, Oklahoma, United States

${ }^{1,2}$ Department of Orthopedic Surgery, Kasr Al-Ainy School of Medicine, Cairo University, Cairo, Egypt

2,4,5,8,9 Limb Lengthening and Complex Reconstruction Service, Department of Orthopedic Surgery, Hospital for Special Surgery, Weill Cornell Medical College, Cornell University, New York, United States

3,7Department of Radiology and Imaging, Hospital for Special Surgery, Weill Cornell Medical College, Cornell University, New York, United States

${ }^{4}$ Saint George's University Medical School, Grenada, West Indies

${ }^{6}$ Department of Clinical Data Core, Healthcare Research Institute, Hospital for Special Surgery, New York, United States

Corresponding Author: S Robert Rozbruch, Limb Lengthening and Complex Reconstruction Service, Department of Orthopedic Surgery, Hospital for Special Surgery, Weill Cornell Medical College, Cornell University, New York, United States, Phone: +1 212 6061415, e-mail: rozbruchsr@hss.edu

How to cite this article: Haleem AM, Galal S, Nwawka OK, et al. Shortterm Results of Magnetic Resonance Imaging after Ankle Distraction Arthroplasty. Strategies Trauma Limb Reconstr 2020;15(3):157-162.

Source of support: Nil

Conflict of interest: None

articular surfaces; improving the healing capacity of articular chondrocytes by creating intermittent fluid pressure; up-regulating proteoglycan metabolism in articular cartilage chondrocytes; 
reducing subchondral bone density by reducing the transmitted forces across the articular cartilage; decreasing synovial membrane inflammation; enhancing the formation of intra-articular fibrocartilage; and stretching the contracted joint capsule. ${ }^{11,14-16}$

A number of studies have reported on clinical and radiographic outcomes of distraction arthroplasty in weight-bearing joints, ${ }^{17-19}$ but there are only a few which have used sophisticated imaging modalities such as $\mathrm{CT}^{20}$ and $\mathrm{MRI}^{21}$ for objective evaluation of the cartilage and subchondral bone. However, none of the studies utilising MRI have incorporated cartilage-specific sequences or objective MRI scoring systems of articular cartilage to report on the outcomes of ankle distraction.

The aim of this study was to examine for positive changes on MRI one year after ankle distraction arthroplasty in addition to radiological findings. The secondary aim of this study was to confirm whether ankle distraction arthroplasty has positive effects on postoperative joint ROM and patient-reported outcomes compared to preoperative levels.

\section{Materials and Methods}

This retrospective case series study was approved by the Institutional Review Board at the investigators' institution.

A cohort of patients who had undergone ankle distraction arthroplasty for unilateral Kellgren-Lawrence grade 3 or 4 ankle osteoarthritis $(\mathrm{OA})^{22}$ with a minimum follow-up of one year were identified from the medical records of the two most senior authors' practices and reviewed retrospectively. During the period from January 1, 2000, to July 31, 2012, a total of 328 patients were identified. Out of this group, a subgroup of 32 consecutive patients (15 males and 17 females, mean age $48.97 \pm 12.75$ years) had undergone preoperative and one-year postoperative MRIs of the ankle joint from January 2010 to October 2012. These paired studies were done at the same institution using a uniform MRI technique and were evaluated by two board-certified musculoskeletal radiologists. MRIs were performed at one-year postoperatively as this coincided with the time of maximal clinical improvement as observed by the two most senior authors. Inclusion criteria were patients between 18 and 70 years with end-stage ankle arthritis. This is defined as incapacitating ankle pain that severely limits activities of daily living and with less than $2 \mathrm{~mm}$ joint space on standard ankle weight-bearing radiographs. The etiology of the arthritis was post-traumatic in all 32 patients. Other inclusion criteria were: ankle mobility (greater than 20 degrees of total ankle arc of motion); absence of or minimal arthritis in adjacent hind foot joints; failure of conservative treatment for a minimum of 6 months; patient's refusal to undergo an ankle arthrodesis; the absence of mental or physical comorbidities or reading disabilities that would prevent them from answering functional questionnaires or obtaining radiographs and MRIs. Contraindications were, limited ankle mobility (less than 20 degrees), patients with severely distorted incongruent articular surfaces (e.g., a flat-top talus), adjacent hind foot arthritis and an inability to comply with postoperative llizarov fixatorr rehabilitation protocol. None of the patients in this subgroup presented with end-stage arthritis due to an inflammatory arthropathy.

\section{Surgical Technique}

The surgical technique follows a standard approach reported previously. ${ }^{10}$ Under spinal-epidural anaesthesia and tourniquet, an arthrotomy was performed via a standard anterior approach. Resection of anterior osteophytes from the tibial and talar sides that caused bony impingement and block to ankle extension was carried out. After complete visualisation and assessment of the articular surfaces, subchondral drilling was performed in areas of eburnated bone. Ankle equinus or a tight gastrocnemius-soleus complex, as evidenced by a positive Silfverskiöld test on preoperative clinical examination, was managed with a concomitant gastrocnemius recession or a gastro-soleus recession (Vulpius) in 26 out of the 32 cases through a posteromedial or direct posterior surgical approach, respectively. ${ }^{23}$ Closure of the surgical incision in anatomical layers and release of tourniquet were followed. Application of the ring external fixator was performed under fluoroscopic guidance using a distal tibial ring and a foot ring with articulating hinges placed along the ankle joint axis between the rings. ${ }^{24}$ Sixty milliliters of bone marrow aspirate (BMA) from the ipsilateral iliac crest obtained at the beginning of the procedure under complete sterile aseptic technique and processed in a commercially available system (Harvest ${ }^{\oplus}$, Terumo BCT, CO, USA) into $7 \mathrm{~mL}$ was injected into the ankle joint under fluoroscopic guidance. Sterile dressings were applied to the surgical site and frame pin sites.

\section{Radiological Outcomes}

Standard weight-bearing X-rays of the ankle (AP, lateral, mortise \pm Saltzman heel views) were obtained. Ankle joint space was measured on all preoperative and postoperative radiographs ${ }^{25}$ by averaging the tibiotalar joint space measurements taken on the anterior-most, middle, and posterior-most aspects of the ankle joint in both AP and lateral views using the picture archiving and communication system (PACS, Philips Easy Vision Healthcare, Bothell, WA, USA). All radiographic measurements were performed by a foot and ankle fellowship-trained orthopedic surgeon.

MRI of the ankle joint was obtained preoperatively and postoperatively for all 32 patients. These images were acquired with an either 1.5 or 3.0 Tesla whole-body scanner (GE Healthcare, Waukesha, WI, USA) using a commercial circumferential ankle coil. Imaging sequences included axial, sagittal, and coronal proton density (PD) and sagittal inversion recovery. The images were retrospectively evaluated by two independent fellowship-trained, board-certified musculoskeletal radiologists on standard PACS workstations. MRIs of the ankle were assessed via a grading system adopted from the knee OA whole-organ magnetic resonance imaging score (WORMS) ${ }^{26}$ and modified to the ankle joint. This grading system included the evaluation of nine parameters: articular cartilage morphology, subchondral bone edema, subchondral cysts size, subchondral cyst number, deformity, subchondral sclerosis, subchondral osteophytes, joint osteophytes, and joint effusion.

In order to localise articular cartilage morphology, the tibial and talar articular surfaces were each divided into four quadrants: anteromedial (AM), posteromedial (PM), anterolateral ( $A L)$, and posterolateral $(\mathrm{PL})$. The articular cartilage was graded on a score of 1-6: 1, normal thickness but increased signal on PD images; 2 , partial or full thickness focal defect $<1 \mathrm{~cm}$ in greatest width; 3 , multiple areas of partial-thickness defects intermixed with areas of normal thickness, or a partial thickness defect wider than $1 \mathrm{~cm}$ but $<75 \%$ of the region; 4 , diffuse ( $\geq 75 \%$ of the region) partial-thickness loss; 5 , multiple areas of full thickness loss or a full thickness lesion wider than $1 \mathrm{~cm}$ but $<75 \%$ of the region; and 6 , diffuse $(\geq 75 \%$ of the region) full-thickness loss.

Subchondral bone marrow edema or abnormality or both were defined as foci of high signal intensity with poorly demarcated margins within the areas of normal fatty marrow on the fat- 
suppressed, fluid-sensitive inversion recovery images. These were graded as 0 , absent; 1 , mild ( $<25 \%$ of the region); 2 , moderate ( $25-50 \%$ of the region); 3 , severe ( $>50 \%$ of the region).

Subchondral cysts were defined as foci of high signal intensity in the subarticular bone with sharply demarcated margins and no evidence of internal marrow tissue or trabecular bone on the fat-suppressed fluid-sensitive images. Cysts were measured in millimeters. The subchondral cyst number was quantified from 0 to -2 or multiple (3 or more) cysts.

A deformity was defined as flattening or depression of the articular surfaces and graded from 0 to 3 based on the subjective degree of deviation from the normal contour: 0 , normal; 1 , mild; 2 , moderate; and 3, severe.

Subchondral sclerosis was defined as follows: 0 , normal; 1 , mild; 2, moderate; and 3, severe. Subchondral osteophytes were graded as present (+) or absent (-). Joint osteophytes along the different margins of the tibia and talus were identified anteriorly, centrally, posteriorly, medially, and laterally. These were graded as follows: 0 , none; 1 , equivocal or small; 2 , medium; and 3, large or very large.

Joint effusion was graded in terms of the estimated maximal distention of the synovial cavity: 0 , normal; $1,<33 \%$ of maximum potential distention; $2,33-66 \%$ of maximum potential distention; and $3,>66 \%$ of maximum potential distention.

The final modified ankle WORMS scores were tabulated as (1) independent values for each feature and (2) cumulative scores for each feature throughout the ankle.

\section{Clinical and Functional Outcomes}

ROM of the operated ankle preoperatively and postoperatively was objectively assessed by the more senior authors using goniometers on all pre- and postoperative visits. This data was extracted from chart review of the latest follow-up visit. In addition, a non-validated three-item questionnaire was administered to all patients as part of standard of care during their latest follow-up visit. This simple questionnaire was used as a subjective guide to clinical outcomes of the ankle distraction procedure. The three questions were:

1. Are you pleased with the outcome of your surgery?

2. Would you do this surgery again?

3. How would you describe your overall functioning after the surgery compared to before surgery: same, better, or worse?

All radiographic measurements, as well as the final subjective and objective data, were plotted on an Excel worksheet (Microsoft, Redmond, WA, USA).

\section{Statistical Analysis}

For statistical analyses, the nonparametric signed-rank test was used due to the small sample size. Statistical analysis was performed on all radiographic measurements to detect difference in joint space on plain X-rays as well as change in each MRI category from pre- to post-operative. Finally, statistical analysis was performed to detect difference in ROM at the same time points. Significance was set at $p<0.05$. Analyses were performed using SAS software version 9.3 (SAS Institute, Inc., Cary, NC, USA).

\section{Results}

The MRI findings are summarised in Table 1. The mean time of fixator removal was $12 \pm 1.8$ weeks. Postoperative radiographs and MRIs were obtained at a mean of $15.67 \pm 6.27$ months after surgery. There was no statistically significant difference between preoperative and post-operative modified WORMSs in terms of cartilage morphology in all four quadrants on both tibial and talar surfaces. There was a trend towards a slight improvement in cartilage morphology in the AM and AL quadrants of both tibial and talar surfaces (Fig. 1) as indicated by the negative change in scores. In addition, a statistically significant reduction in joint osteophytes was noticed post-operatively ( $p=0.0129)$. There was no statistically significant difference post-operatively in the subcategories of bone marrow edema, cyst size and count, articular surface deformity, subchondral sclerosis, and subchondral osteophytes. Unexpectedly, there was a statistically significant increase in joint effusion post-operatively compared to preoperative levels ( $p=0.03$ ) (Table 1). Figures 1 and 2 represent examples of cases with highest and lowest WORMS scores, respectively.

There was a statistically significant difference between preoperative ankle joint space measured on X-rays of both AP (mean $1.66 \pm 1.12 \mathrm{~mm}$ ) and lateral views (mean $1.65 \pm 1.00 \mathrm{~mm}$ ) compared to post-operative AP (mean $2.78 \pm 1.07 \mathrm{~mm}$ ) and lateral (mean $2.91 \pm 1.14 \mathrm{~mm}$ ) films. This difference was significant in both AP (mean $1.11 \pm 0.90 \mathrm{~mm}$ ) and lateral (mean $1.25 \pm 0.84 \mathrm{~mm}$ ) views $(p<0.0001)$.

The mean time to latest follow-up for postoperative assessment of ankle ROM and administering the three-item questionnaire was $19.60 \pm 9.93$ months. The mean preoperative vs postoperative ankle dorsiflexion was $1.97 \pm 13.42^{\circ}$ vs $7.34 \pm 7.93^{\circ}$, respectively. This translated into a statistically significant postoperative gain in ankle dorsiflexion of $5.38 \pm 7.40^{\circ}(p<0.0001)$. On the other hand, plantarflexion was not significantly impacted. The mean preoperative vs postoperative ankle plantarflexion was $42.97 \pm 9.90^{\circ}$ vs $40.31 \pm 10.54^{\circ}$, respectively. There was a mean postoperative loss in ankle plantarflexion of $2.66 \pm 10.39^{\circ}$, which was not statistically significant $(p<0.29)$. The total ankle arc of

Table 1: Pre-operative to post-operative changes in MRI subcategories after ankle distraction arthroplasty according to the modified WORMS score. Negative values in the "cartilage" subcategory indicate improvement. $p$-values $<0.05$ are marked by $\left(^{*}\right)$

\begin{tabular}{llcll}
\hline Variable & Values & Meanchange & Std dev & $p$-value \\
\hline Tibia AM & Cartilage (1-6) & -0.39286 & 1.25725 & 0.424 \\
Tibia PM & & 0.17857 & 1.0203 & 0.6291 \\
Tibia AL & & -0.28571 & 1.01314 & 0.3018 \\
Tibia PL & & -0.25 & 0.96705 & 0.6072 \\
Talus AM & Cartilage (1-6) & -0.03571 & 0.99934 & 1 \\
Talus PM & & 0.32143 & 1.27812 & 0.6291 \\
Talus AL & & -0.07143 & 1.08623 & 1 \\
Talus PL & & 0.10714 & 1.03062 & 0.6291 \\
Edema & $0-3$ & 0.14286 & 1.0789 & 0.6291 \\
Cyst size & mm & 0.05 & 3.88621 & 0.8145 \\
Cyst ount & $0,1,2, \mathrm{~m}$ & 0.03571 & 1.50264 & 1 \\
Deformity & $0-3$ & 0.35714 & 0.86984 & 0.1185 \\
Sclerosis & $0-3$ & 0.07407 & 0.82862 & 0.7744 \\
OP & $0-3$ & -0.44444 & 0.75107 & $0.0129^{*}$ \\
Effusion & $0-3$ & 0.42857 & 0.9595 & $0.0309^{*}$ \\
SC OP & Present/absent & & & 0.2891 \\
\hline AM, anterom
\end{tabular}

$\mathrm{AM}$, anteromedial; PM, posteromedial; $\mathrm{AL}$, anterolateral; $\mathrm{PL}$, posterolateral; $\mathrm{mm}$, millimeter; $\mathrm{m}$, multiple; OP, joint osteophytes; SC OP, subchondral osteophytes 

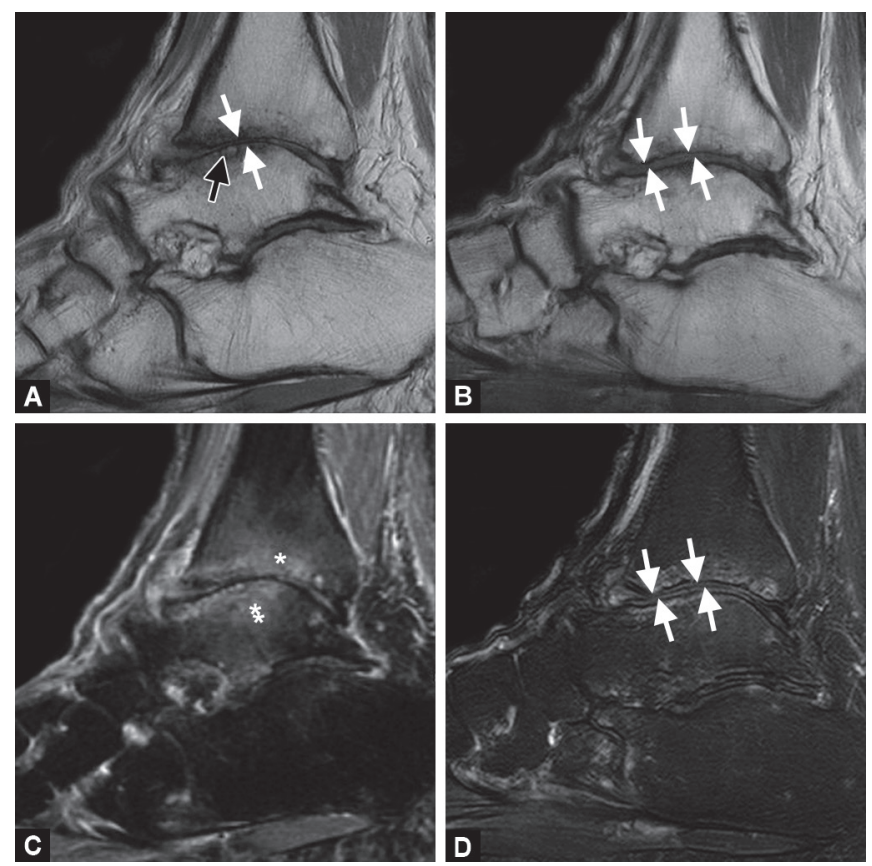

Figs $1 \mathrm{~A}$ to $\mathrm{D}$ : Example of a high WORMS grade ankle post-distraction: Sagittal PD (A, B) and fat-suppressed (C, D) MRI images of the ankle joint in a 64-year-old woman undergoing ankle distraction. Pre-distraction images $(A, C)$ reveal full-thickness cartilage loss over the tibia and talus (A, white arrows) with joint space narrowing and subchondral cyst formation ( $A$, black arrow). There is corresponding severe, reactive bone marrow edema pattern on both sides of the joint $(C, *)$. Post-distraction images $(B, D)$ demonstrate diffuse fill-in of presumed fibrocartilagenous material over the tibia and talus widening the joint space $(B, D$, white arrows), as well as decreased bone marrow edema pattern

motion tended to improve but this was not significantly different statistically from preoperative $\left(44.94 \pm 14.03^{\circ}\right)$ to postoperative $\left(47.81 \pm 12.82^{\circ}\right)$ measurements $(p=0.05)$.

Female patients in this cohort were significantly older than males (53.24 \pm 14.77 years vs $44.13 \pm 7.99$ years, respectively) and had significantly higher plantarflexion limits compared to males preoperatively $\left(45.88 \pm 8.88^{\circ}\right.$ vs $\left.39.67 \pm 10.26^{\circ}\right)$ and postoperatively $\left(43.24 \pm 10.3^{\circ}\right.$ vs $\left.37.0 \pm 10.14^{\circ}\right)(p=0.025$ and $p=0.04$, respectively).

Results from the item-questionnaire administered at the latest follow-up postoperative visit indicated that $87.5 \%$ of patients were pleased with the outcome of surgery and $84.3 \%$ mentioned they would have the surgery again. In terms of function, $62.5 \%$ of patients were functioning significantly better after the surgery, $31.2 \%$ were functioning the same, and $6.25 \%$ were functioning better before the surgery. Complications included superficial pin tract infections in $80 \%$ of patients, which were managed with a single course of oral antibiotics. No patients developed deep joint infections or septic arthritis. There were no cases or neurovascular injury, RSD, arthrofibrosis, or peri-articular fractures.

\section{Discussion}

The aim of this study was to examine the positive changes on MRI one year after ankle distraction arthroplasty in addition to radiological findings. The secondary aim of this study was to confirm if ankle distraction arthroplasty has positive effects on
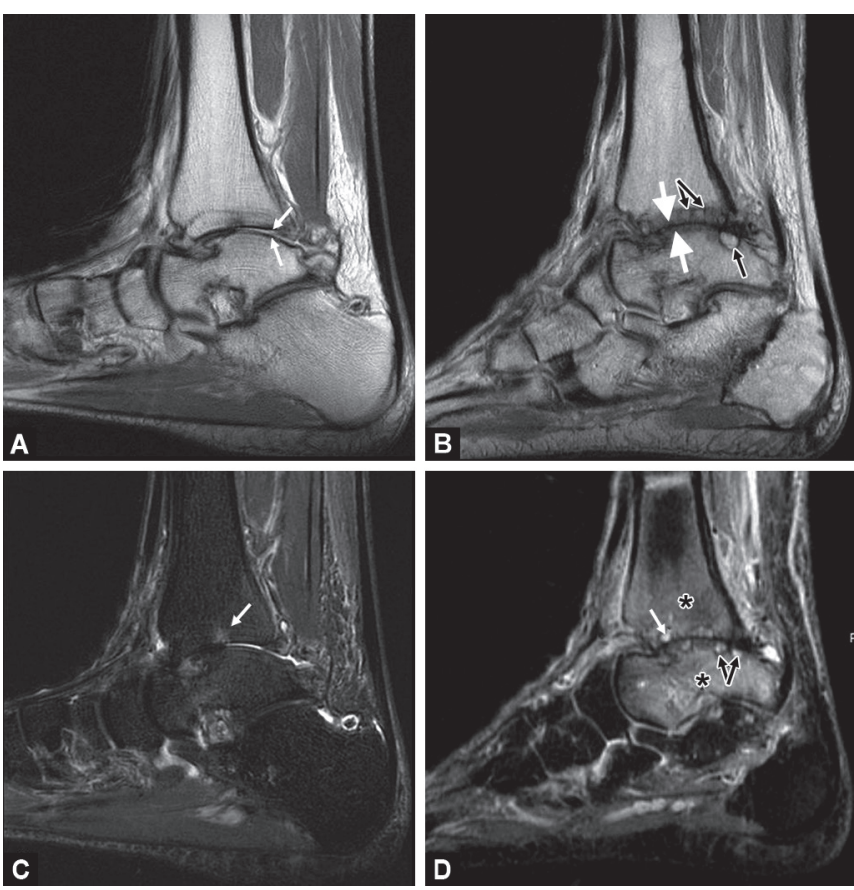

Figs. 2A to D: Example of a lowWORMS score ankle post-distraction: Sagittal PD (A, B) and fat-suppressed (C, D) MRl images of the ankle joint in a 62-yearold woman undergoing ankle distraction. Pre-distraction images $(A, C)$ reveal full-thickness cartilage loss at the ankle, worse over the posterior tibia and talus ( $\mathrm{A}$, white arrows). There is corresponding mild, reactive bone marrow edema pattern on the tibial side ( $C$, white arrow). Post-distraction images ( $\mathrm{B}_{\text {, }}$ D) demonstrate marked progression in oseteoarthritis, with diffuse cartilage loss, obliteration of the joint space ( $B$, white block arrows), and prominent subchondral cysts ( $B, D$, black arrows). The corresponding fat suppressed image reveals severe subchondral bone marrow edema pattern $\left(D,{ }^{*}\right)$

postoperative joint ROM and patient-reported outcomes compared to preoperative levels.

Three questions need to be answered:

1. Is there a positive change on MRI after ankle distraction?

2. Is there an improvement on X-ray and in joint ROM after ankle distraction?

3. What is the patient-reported outcome after treatment?

This is the largest case series in the literature at present comparing preoperative and postoperative MRI findings of ankle distraction arthroplasty. An objective scoring system for the grading of such MRI changes, as interpreted by independent musculoskeletal radiologists who had expertise in this modality, was used. The modified (WORMS) score offers potential for multi-feature assessment of the ankle using conventional MRI. It takes into account a variety of features believed to be relevant to the functional integrity of weight-bearing joints and the pathophysiology of OA. It scores each of these features with sufficient increments to allow detection of what are speculated to be clinically relevant changes. WORMS scores employ conventional MR images that can be produced with MRI systems available at most hospitals. There is high inter-reader agreement among trained radiologists experienced in musculoskeletal MRI interpretation. ${ }^{26}$ The modified WORMS score employed in this study has not been validated for ankle OA but, at the time of initiation of this study, no other objective MRI scoring system has 
been for the quantification of postoperative MRI changes in the arthritic ankle after salvage procedures.

Our results did not show significant improvement in any of the subcategories tested on MRI except for joint osteophytes. The significant reduction in joint osteophytes is clearly attributable to their complete excision intraoperatively. An unexpected significant increase in joint effusion postoperatively was noted. It is not clear why this occurred; although it could be attributed to a persistent inflammatory or healing response at this early follow-up period. In the subcategory of cartilage morphology, the anterior quadrants of both tibial and talar surfaces showed slightly better modified WORMS scores postoperatively. It is thought that the anterior half of the tibiotalar joint is the part most exposed through the arthrotomy by the anterior surgical approach and that the preferential debridement and microfracture of the more accessible anterior half of the joint compared to the relatively inaccessible posterior half may be responsible. The non-significant improvement in cartilage morphology could be attributed to immaturity of the reparative tissue at this early follow-up phase with incomplete resolution of marrow changes. However, this tissue did not exhibit the characteristic grayscale stratification on MRI consistent with hyaline cartilage, but rather diffuse high signal as seen with fibrous tissue or fibrocartilage. A prior case report of ankle arthroscopy following ankle distraction demonstrated fill of the joint with a layer of fibrocartilage-like tissue. ${ }^{27}$ Finally, the subchondral bone did not show improvement or normalization in signal postoperatively. This contrasts with other studies where Intema et al. ${ }^{20}$ utilised CT to evaluate ankle distraction arthroplasty and reported on an overall decreased density in subchondral sclerotic areas together with normalisation of bone density in cystic lesions. They found no positive correlations between a globally diminished sclerosis and clinical improvement, similar to the findings in this study. In two different preliminary reports of three cases each, Lamm et al. ${ }^{21}$ and Van Meegeren et al. ${ }^{28}$ reported improved MRI findings after ankle distraction in post-traumatic arthritis and hemophilic arthropathy, respectively. Subchondral bone edema and effusion seemed to deteriorate postoperatively on the MRIs in Lamm's case series. ${ }^{21}$ These positive findings in both case series were limited to diminution of subchondral bone cysts only, with cartilage thicknesses varying between an increase and decrease in hemophilic ankles ${ }^{28}$ and increasing an average of $0.5 \mathrm{~mm}$ in post-traumatic arthritis. ${ }^{21}$ The insufficient number of patients in both of these studies with insufficient data on the method of MRI grading does not aid in drawing any conclusions. Wiegant et al. ${ }^{12}$ quantified their MRI findings after knee distraction arthroplasty, demonstrating a significant increase in cartilage thickness 2 years postoperatively, together with increased joint space and decreased denuded subchondral bone. The MRI sequence used to quantify cartilage thickness in this study was a 3D-spoiled gradient recalled (SPGR) sequence with fat suppression, which does not distinguish between joint fluid and the adjacent cartilaginous interface, potentially giving a false reading of increased cartilage thickness on the expense of an expanding joint space and synovial fluid.

Radiographic measurements showed a gain in ankle joint space of $1.19 \pm 0.79 \mathrm{~mm}$ on both AP and lateral views. Our findings concur with other studies that have demonstrated improvement in joint space or radiographic symptoms of OA or both. ${ }^{7}$ Lee et al. reported a mean increase in radiographic joint space of $2 \mathrm{~mm}$ in a case series of ankle distraction augmented with allograft collagen membrane. ${ }^{29}$ The senior authors are diligent about obtaining a minimum of $5.8 \mathrm{~mm}$ increase in joint space during ankle distraction to effectively unload the ankle joint. ${ }^{5}$ Postoperative $X$-rays revealed a gradual decline in joint space on weight-bearing ankle X-rays but without any deterioration in symptoms as noted from the clinical functional questionnaires administered at the same time point. This is again, in concurrence with results of other studies, as those reported by Paley et al. ${ }^{30}$

The results in this study demonstrate that while ankle distraction resulted in a significant gain of dorsiflexion, it did not completely normalise ROM. This is in agreement with other studies, which have showed that ankle ROM after distraction arthroplasty was maintained at a similar level, ${ }^{10,30}$ or sometimes with an insignificant increase. ${ }^{8}$ It is to be noted that the increase in ankle ROM observed in this study could be partially attributed to auxiliary procedures performed at the time of ankle distraction, namely anterior osteophyte resection and gastric-soleus recession. Despite this, it is possible to perform most activities of daily living with a limited ROM of the ankle because most of these activities require only partial $\mathrm{ROM}^{31}$

The results of the current study demonstrate an overall satisfaction rate of $87 \%$ after ankle distraction with $67 \%$ patients noting functional improvement postoperatively compared to preoperative levels. This is again in agreement with most studies that have reported similar satisfaction rates, ranging from 73 to $91 \%$, in functional improvement as well as pain reduction. ${ }^{29,30}$ While some studies utilise AOFAS scores in evaluation of ankle distraction, it is worth mentioning that the AOFAS score has not been validated for ankle arthritis and distraction arthroplasty. The idea behind using a three-item questionnaire in this current study was to assess patient's general satisfaction and functionality in a simple, cost- and time-efficient manner at their latest follow-up visit.

There are a number of limitations to this study. The retrospective nature with a limited number of patients might account for the lack of significant difference in WORMS scores between preoperative and postoperative imaging. Another potential reason for the lack of significant differences in WORMS scores is that the scoring system may not be sensitive enough for changes in cartilage. If there is cartilage regeneration, with continued moderate thinning, the score may not change. This study has a short-term mean follow-up period of one year for postoperative MRIs. The reduction in osteophytes, originally the result of direct osteophyte resection at the time of surgery but still absent at one year, should be interpreted with caution due to the short-term follow-up. Longer-term follow-up studies are further required to validate this finding. One of the nine parameters of the modified WORMS scores included reporting on joint and subchondral osteophytes, which opted us to keep it in the modified ankle version of this score reported in our study.

The WORMS is validated for knee OA but not for ankle OA. Due to the absence of any validated MRI scoring systems for ankle OA, WORMS was deemed the most appropriate to report. The interpretation of a non-validated three-item questionnaire for the assessment of patient-reported outcomes needs caution in that patients tend to describe worse pain and physical function preoperatively on retrospective questioning, especially when they are satisfied with the outcome of surgery. As such, the improvement in clinical status in our study might be overestimated.

\section{CONCLUSION}

The current study demonstrates that MRI results at one-year postankle distraction arthroplasty do not reveal statistically significant improvements in most of the ankle arthritis parameters through 
utilising the modified WORMS. Despite these MRI findings, there were significant improvements in the radiographic joint space, functional outcomes, and maintenance of ankle ROM notably a significant increase in dorsiflexion. Further studies with a larger number of patients and longer follow-up periods are needed. A validation and refinement of WORMS for the arthritic ankle joint are needed as well to maximise MRI as a utility for assessing ankle $\mathrm{OA}$ and changes before and after intervention.

\section{References}

1. Fuchs S, Sandmann C, Skwara A, et al. Quality of life 20 years after arthrodesis of the ankle. A study of adjacent joints. J Bone Joint Surg Br 2003;85(7):994-998. DOI:10.1302/0301-620x.85b7.13984.

2. Thomas R, Daniels TR, Parker K. Gait analysis and functional outcomes following ankle arthrodesis for isolated ankle arthritis. J Bone Joint Surg Am 2006;88(3):526-535. DOI:10.2106/JBJS.E.00521.

3. Jastifer JR, Coughlin MJ. Long-term follow-up of mobile bearing total ankle arthroplasty in the United States. Foot Ankle Int 2014;36(2):143150. DOI: $10.1177 / 1071100714550654$.

4. de Asla RJ, Ellis S, Overley B, et al. Total ankle arthroplasty in the setting of valgus deformity. Foot Ankle Spec 2014;7(5):398-402. DOI: 10.1177/1938640014549535.

5. Brunner S, Barg A, Knupp M, et al. The Scandinavian total ankle replacement: long-term, eleven to fifteen-year, survivorship analysis of the prosthesis in seventy-two consecutive patients. J Bone Joint Surg Am 2013;95(8):711-718. DOI: 10.2106/JBJS.K.01580.

6. Kraal T, van der Heide HJ, van Poppel BJ, et al. Long-term follow-up of mobile-bearing total ankle replacement in patients with inflammatory joint disease. Bone Joint J 2013;95-B(12):1656-1661. DOI: 10.1302/0301-620X.95B12.32146.

7. van Valburg AA, van Roermund PM, Lammens J, et al. Can Ilizarov joint distraction delay the need for an arthrodesis of the ankle? A preliminary report. J Bone Joint Surg Br 1995;77(5):720-725.

8. van Valburg AA, van Roermund PM, Marijnissen AC, et al. Joint distraction in treatment of osteoarthritis: a two-year follow-up of the ankle. Osteoarthritis Cartilage 1999;7(5):474-479.

9. Marijnissen AC, Hoekstra MC, Pre BC, et al. Patient characteristics as predictors of clinical outcome of distraction in treatment of severe ankle osteoarthritis. J Orthop Res 2014;32(1):96-101. DOI: 10.1002/ jor.22475.

10. Tellisi N, Fragomen AT, Kleinman D, et al. Joint preservation of the osteoarthritic ankle using distraction arthroplasty. Foot Ankle Int 2009;30(4):318-325. DOI: 10.3113/FAI.2009.0318.

11. van Valburg $A A$, van Roermund $P M$, Marijnissen $A C$, et al. Joint distraction in treatment of osteoarthritis (II): effects on cartilage in a canine model. Osteoarthritis Cartilage 2000;8(1):1-8. DOI: 10.1053/ joca.1999.0263.

12. Wiegant $K$, van Roermund PM, Intema F, et al. Sustained clinical and structural benefit after joint distraction in the treatment of severe knee osteoarthritis. Osteoarthritis Cartilage 2013;21(11):1660-1667. DOI: 10.1016/j.joca.2013.08.006

13. Yanai T, Ishii T, Chang F, et al. Repair of large full-thickness articular cartilage defects in the rabbit: the effects of joint distraction and autologous bone-marrow-derived mesenchymal cell transplantation. J Bone Joint Surg Br 2005;87(5):721-729. DOI: 10.1302/0301620X.87B5.15542.

14. Morse KR, Flemister AS, Baumhauer JF, et al. Distraction arthroplasty. Foot Ankle Clin 2007;12(1):29-39. DOI: 10.1016/j.fcl.2006.11.003.
15. van Roermund PM, Marijnissen AC, Lafeber FP. Joint distraction as an alternative for the treatment of osteoarthritis. Foot Ankle Clin 2002;7(3):515-527. DOI: 10.1016/S1083-7515(02)00027-X.

16. van Valburg AA, van Roy HL, Lafeber FP, et al. Beneficial effects of intermittent fluid pressure of low physiological magnitude on cartilage and inflammation in osteoarthritis. An in vitro study. J Rheumatol 1998;25(3):515-520.

17. Aldegheri R, Trivella G, Saleh M. Articulated distraction of the hip. Conservative surgery for arthritis in young patients. Clin Orthop Relat Res 1994(301):94-101.

18. Marijnissen AC, Van Roermund PM, Van Melkebeek J, et al. Clinical benefit of joint distraction in the treatment of severe osteoarthritis of the ankle: proof of concept in an open prospective study and in a randomized controlled study. Arthritis Rheum 2002;46(11):28932902. DOI: 10.1002/art.10612.

19. Ploegmakers JJ, van Roermund PM, van Melkebeek J, et al. Prolonged clinical benefit from joint distraction in the treatment of ankle osteoarthritis. Osteoarthritis Cartilage 2005;13(7):582-588. DOI: 10.1016/j.joca.2005.03.002.

20. Intema F, Thomas TP, Anderson DD, et al. Subchondral bone remodeling is related to clinical improvement after joint distraction in the treatment of ankle osteoarthritis. Osteoarthritis Cartilage 2011;19(6):668-675. DOI: 10.1016/j.joca.2011.02.005.

21. Lamm BM, Gourdine-Shaw M. MRI evaluation of ankle distraction: a preliminary report. Clin Podiatr Med Surg 2009;26(2):185-191. DOI: 10.1016/j.cpm.2008.12.007.

22. Kellgren JH, Lawrence JS. Radiological assessment of osteo-arthrosis. Ann Rheum Dis 1957;16(4):494-502. DOI: 10.1136/ard.16.4.494.

23. Firth GB, McMullan M, Chin T, et al. Lengthening of the gastrocnemiussoleus complex: an anatomical and biomechanical study in human cadavers. J Bone Joint Surg Am 2013;95(16):1489-1496. DOI: 10.2106/ JBJS.K.01638.

24. Inda DA, Blyakher A, O'Malley MJ, et al. Distraction arthroplasty for the ankle using the llizarov frame. Tech Foot Ankle Surg 2003;2(4):249253. DOI: 10.1097/00132587-200312000-00005.

25. Fragomen AT, McCoy TH, Meyers KN, et al. Minimum distraction gap: how much ankle joint space is enough in ankle distraction arthroplasty? HSS J 2014;10(1):6-12. DOI: 10.1007/s11420013-9359-3.

26. Peterfy CG, Guermazi A, Zaim S, et al. Whole-Organ Magnetic Resonance Imaging Score (WORMS) of the knee in osteoarthritis. Osteoarthritis Cartilage 2004;12(3):177-190. DOI: 10.1016/ j.joca.2003.11.003.

27. Kanbe K, Hasegawa A, Takagishi K, et al. Arthroscopic findings of the joint distraction for the patient with chondrolysis of the ankle. Diagn Ther Endosc 1997;4(2):101-105. DOI: 10.1155/DTE.4.101.

28. Van Meegeren ME, Van Veghel K, De Kleijn P, et al. Joint distraction results in clinical and structural improvement of haemophilic ankle arthropathy: a series of three cases. Haemophilia 2012;18(5):810-817. DOI: 10.1111/j.1365-2516.2012.02805.x.

29. Lee DK. Ankle arthroplasty alternatives with allograft and external fixation: preliminary clinical outcome. J Foot Ankle Surg 2008;47(5):447-452. DOI: 10.1053/j.jfas.2008.04.011.

30. Paley D, Lamm BM, Purohit RM, et al. Distraction arthroplasty of the ankle-how far can you stretch the indications? Foot Ankle Clin 2008;13(3):471-484, ix. DOI: 10.1016/j.fcl.2008.05.001.

31. Sinitski EH, Hansen AH, Wilken JM. Biomechanics of the ankle-foot system during stair ambulation: implications for design of advanced ankle-foot prostheses. J Biomech 2012;45(3):588-594. DOI: 10.1016/j. jbiomech.2011.11.007. 